\title{
Decisões, complexidades, agentes e sunk costs: um estudo comparativo envolvendo gestores com diferentes perfis
}

\author{
Uilcleides Braga da Silva \\ Mestrado em Contabilidade e Controladoria pela Universidade Federal da Bahia - \\ UFBA \\ Rua Auristides, 2. Federação. Salvador/BA. CEP: 40210-340 \\ E-mail: uilcleidesbraga@yahoo.com.br
}

Adriano Leal Bruni

Doutorado em Administração pela Universidade de São Paulo - USP

Professor Titular da Universidade Federal da Bahia - UFBA

Rua Auristides, 2. Federação. Salvador/BA. CEP: 40210-340

E-mail: albruni@gmail.com

José Maria Dias Filho

Doutorado em Contabilidade e Controladoria pela Universidade de São Paulo - USP

Professor Adjunto da Universidade Federal da Bahia - UFBA

Rua Auristides, 2. Federação. Salvador/BA. CEP: 40210-340

E-mail:zemariadias@uol.com.br

Enoque Barbosa dos Santos Mestrando em Contabilidade pela Universidade Federal da Bahia - UFBA

Professor da Universidade Federal da Bahia - UFBA

Rua Auristides, 2. Federação. Salvador/BA. CEP: 40210-340

E-mail: enoqb@hotmail.com

\section{RESUMO}

Esse estudo tem como objetivo verificar a ocorrência de vieses cognitivos associados ao sunk cost (o viés sunk cost se manifesta quando um individuo apresenta maior disposição em continuar um investimento, mesmo tendo conhecimento de que os resultados ou retornos podem estar comprometidos somente pelo simples fato de já ter iniciado e aplicado algum recurso no projeto), considerando possíveis características dos respondentes como: tempo de experiência profissional e em cargos de gestão; período de vinculação profissional a entidades públicas ou privadas, bem como idade e gênero. Foi constituído um quase-experimento envolvendo situações com a ocorrência de sunk cost, contando com a participação de 128 alunos de cursos de pós-graduação de Instituições de Ensino Superior de Salvador, Bahia. As análises foram feitas com o uso de testes Qui-quadrado e teste paramétrico de igualdade de médias para verificar as diferenças das respostas, bem como associação do viés e outras variáveis. Os resultados da pesquisa indicaram a manifestação do viés relativo ao sunk cost. Contudo, não foi possível explicar a sua ocorrência a partir das variáveis. O fato de uma pessoas ser mais experiente em cargos de gestão não influencia na ocorrência do viés, 
Decisões, complexidades, agentes e sunk costs: um estudo comparativo envolvendo gestores com diferentes perfis.

Uilcleides Braga da Silva, Adriano Leal Bruni, José Maria Dias Filho, Enoque Barbosa dos Santos

bem como trabalhar em instituições que objetivam lucro como as entidades do setor privado, que a princípio esperava-se que tivesse impacto na ocorrência do viés, devido a pressões por parte dos proprietários a ter resultados positivos que garantam retornos do capital investido e a continuidade da empresa. Outras variáveis como idade e gênero também não influenciaram de forma significativa na ocorrência do fenômeno.

Palavras-Chave: Decisões. Efeito Sunk Cost. Finanças Comportamentais.

\section{Decisions, complexities, agents and sunk costs: a comparative study involving managers with different profiles}

\section{ABSTRACT}

The present study aims verify the occurrence of cognitive biases associated with the sunk cost. (sunk cost bias manifests itself when an individual has a higher willingness to continue an investment, even knowing that the results or returns can be committed only by the mere fact of having begun and applied some resource in the project), investigating possible characteristics of the respondent as: time and experience in management positions; period linking professional public or private entities, as well as age and gender. It was constituted a quasi-experiment involving situations with the occurrence of sunk cost, with the participation of 128 students graduate courses in Higher Education Institutions in Salvador, Bahia. The analyzes were performed using chi-square and parametric test for equality of means to verify the differences in the responses and the association of bias and other variables. The results indicate the manifestation of bias on the sunk cost. However it was not possible to explain their occurrence based on the variables. The fact that a person be more experienced in management positions do not influence the occurrence of bias, as well as working in institutions that aim to profit as private sector entities that at first it was expected to impact on the occurrence of bias due to pressure by the owners to have positive results to ensure returns on invested capital and business continuity. Other variables such as age and gender did not influence significantly the occurrence of the phenomenon.

Keywords: Decisions. Sunk Cost Effect. Behavioral Finance.

\section{INTRODUÇÃO}

O processo de tomada de decisão precisa ser analisado sob o ponto de vista da sua complexidade, considerando gestores processando informações limitadas, e a existência de incertezas no ambiente organizacional, que sofre interferências de 
Decisões, complexidades, agentes e sunk costs: um estudo comparativo envolvendo gestores com diferentes perfis.

Uilcleides Braga da Silva, Adriano Leal Bruni, José Maria Dias Filho, Enoque Barbosa dos Santos

ambientes externos a organização. As mudanças estruturais que influenciam diretamente no processo não são explicadas totalmente pelas teorias econômicas, surgindo a necessidade de novas teorias que possam envolver tanto aspectos racionais como comportamentais e sociológicos para tentar explicar esse processo.

Decisões são influenciadas por variáveis internas quanto externas. Como variáveis de dimensão interna temos estrutura organizacional, aspectos financeiros, gestão de pessoas, e outros, e externas, mudanças na economia, aspectos culturais, sociais, que acabam restringindo a racionalidade das pessoas envolvidas e impactando na decisão e ainda variáveis comportamentais característicos da natureza humana.

Tal complexidade pode levar o agente de decisão a fazer uso de meios simplificadores (heurísticas) para sintetizar as informações e fazer seus julgamentos. $O$ uso desses princípios heurísticos não deve ser visto como uma maneira incorreta de julgar, considerando que as informações são incompletas em um ambiente de incerteza e o uso dessas simplificações pode ajudar no processo ao considerar aspectos como experiência, informações culturais, histórico de eventos e sua representatividade de uma determinada atividade, praticam legitimadas pela sociedade como âncora. $\mathrm{O}$ problema está anunciado quando os desvios ou erros de julgamentos são capazes de comprometer o resultado esperado. São os chamados vieses que influenciam de maneira negativa na decisão.

O efeito sunk costs é um desses fenômenos, onde as pessoas apresentam maior disposição em continuar um investimento mesmo sabendo que os resultados/retornos estão comprometidos simplesmente pelo fato de já ter sido investido uma parte do recurso financeiro. Embora seja sugerido que os custos irrecuperáveis que não são relevantes, ou seja, os custos já incorridos não devem ser considerados para decisões de investir ou não um determinado recurso, os estudos no âmbito comportamental e situações práticas provaram o contrário, as pessoas são sensíveis ao sunk cost nos seus julgamentos. Este viés é abordado com detalhes nesse artigo.

Considerando que os vieses cognitivos podem levar a decisões indevidas, esse estudo visa observar o comportamento de tomadores de decisões de dois setores 
Decisões, complexidades, agentes e sunk costs: um estudo comparativo envolvendo gestores com diferentes perfis.

Uilcleides Braga da Silva, Adriano Leal Bruni, José Maria Dias Filho, Enoque Barbosa dos Santos

distintos um com fins lucrativos - setor privado e outro sem fins lucrativos - setor público, partindo do pressuposto de que as entidades que almejam lucro devem estar constantemente preocupadas em obter resultados positivos e, portanto, devem ser mais racionais no momento de decidir seus investimentos. Assim, é relevante observar e tentar entender o comportamento desses gestores em relação ao efeito sunk costs, visto que não foram encontrados estudos com essa abordagem.

No que tange às organizações públicas, no decorrer das duas últimas décadas estas têm passado por grandes transformações em suas políticas de gestão, isso se deve a alguns fatores como "uma forte crise fiscal, o esgotamento do modelo de desenvolvimento que era baseado no financiamento externo, os problemas sociais crescentes, a má distribuição, incapacidade governamental de coordenar e implementar políticas públicas que pudessem, de fato, transformar esse contexto" (VAZ; LOTTA, 2011).

Observando esse ambiente, Vaz (2006) apresenta três forças que direcionaram a evolução do serviço público brasileiro: a) a racionalização do uso de recursos crescentemente escassos; b) a demanda por um novo patamar de qualidade dos serviços, e, c) a pressão da sociedade por participação, transparência e controle social sobre as ações dos agentes públicos.

Em 2000 tem se a edição da Lei de Responsabilidade Fiscal $(2000)^{1}$, um instrumento que objetiva impor o controle dos gastos públicos e promove a transparência dos gastos públicos, ou seja, os gestores públicos são obrigados a prestar contas de maneira detalhada da destinação orçamentária. Os recursos públicos têm um controle maior por parte do próprio governo, sendo este controle exercido de forma legal, ou seja, existem instrumentos legais determinando as regas para a administração dos recursos.

As entidades públicas não visam lucro como ocorre nas empresas do setor privado, estas não devem estar tão preocupadas com os resultados positivos de um

\footnotetext{
${ }^{1}$ Lei de Responsabilidade Fiscal - nº 101/2000: Lei Complementar que estabelece normas de finanças públicas voltadas para a responsabilidade na gestão fiscal.
} 
Decisões, complexidades, agentes e sunk costs: um estudo comparativo envolvendo gestores com diferentes perfis.

Uilcleides Braga da Silva, Adriano Leal Bruni, José Maria Dias Filho, Enoque Barbosa dos Santos

investimento quanto às empresas do setor privado. Estão sim preocupadas com a destinação dos recursos em fase orçamentária, devido a pressões legais e a prestar contas da destinação apenas e não da qualidade positiva ou negativa do resultado do investimento, sendo que resultados negativos de um investimento não ocasionam comprometimento das suas atividades diferentemente das empresas privadas que tem a pressão de seus proprietários por resultados positivos.

Sendo assim, pode se considerar que os profissionais envolvidos no setor privado são menos propensos ao efeito sunk cost, devido à pressão exercida pelos proprietários e exigências de bons resultados, visto que são os lucros que garantem a sua continuidade.

Outro ponto importante a ser observado é a experiência profissional. Acredita-se que uma experiência profissional possa melhorar a compreensão e percepção de forma mais racional dos indivíduos devido ao fato de que a vivencia com o ambiente pode ocasionar alguma confiança no momento de decidir.

Também quando se tem experiência em cargos de gestão é possível que se tenha maior facilidade e tranquilidade em lidar com processos que envolvem decisão de investimentos por apresentar algum grau de confiança. Nesse sentido, Bruni et al. (2010) mencionam que um maior envolvimento dos indivíduos no processo orçamentário aumenta o viés excesso de confiança.

Acredita-se a princípio que a experiência poderia influenciar no julgamento, considerando que o tomador de decisão lida constantemente com opções de investimentos, tendo que tomar decisões e observar os custos e benefícios de suas decisões, este pode estar mais atento à ocorrência de perdas nos investimentos.

Em linha com o exposto, esse estudo investiga a manifestação de vieses cognitivos associados ao sunk cost junto a profissionais com diferentes perfis, examinando a associação entre a manifestação do viés e características dos respondentes como: tempo de experiência profissional; experiência profissional em cargos de gestão; tempo de vinculação profissional a entidades públicas ou privadas. Adicionalmente são controlados aspectos como idade e gênero. 
Decisões, complexidades, agentes e sunk costs: um estudo comparativo envolvendo gestores com diferentes perfis. Uilcleides Braga da Silva, Adriano Leal Bruni, José Maria Dias Filho, Enoque Barbosa dos Santos

\section{FUNDAMENTAÇÃO TEÓRICA}

A abordagem clássica da economia sugere que as decisões são tomadas considerando escolha racional numa perspectiva de eficiência de mercado considerando a inexistência de informações incompletas ou imperfeitas, observando a melhor opção que permita a maximização dos retornos marginais.

A teoria da utilidade esperada preconiza a escolha sob perspectiva da objetividade, onde as decisões são tomadas com base em informações completas, desconsiderando o fato da existência de situações de risco e pressões de âmbito comportamental e temporal, ou seja, as decisões a luz dessa teoria são tomadas considerando apenas a racionalidade. (CAVALHEIRO, 2010).

As pesquisas internacionais de Simon, Jones, Kahneman, Tversky, Barberis, Shleifer e Vishny, Rose e Rose, Hobson e Kachelmeier, Springer e Borthick e outros, indicam que a teoria da utilidade esperada apresenta anomalias, e que os indivíduos tomam suas decisões considerando não somente aspectos lógicos, como também aspectos comportamentais. Observam que as pessoas que apresentam maior sensibilidade a perdas do que a resultados positivos, também apresentam maior sensibilidade nas escolhas quando se alteram os riscos, tentam simplificar ao máximo uma situação, o que pode levar a decisões desvirtuadas da realidade, conforme definição da Teoria dos Prospectos (Prospect Theory) abordada por Kahneman e Tversky (WATT; VÁZQUEZ, 2012).

As pesquisas de Tversky e Kahneman divulgadas em seu artigo "Judgment under Uncertainty: Heuristics and Biases" são precursoras dos estudos comportamentais na década de 1970, descrevendo as principais heurísticas utilizadas para avaliar probabilidades e predizer valores: Representatividade, Disponibilidade e Ancoragem.

$\mathrm{Na}$ mesma direção, Silva e Lima (2007), Domingos (2007), Bruni et al. (2010), Segantini, Vieira e Silva (2011), desenvolveram trabalhos empíricos contestando a validade dessa teoria. Em seus achados demonstram evidencias de outras variáveis 
Decisões, complexidades, agentes e sunk costs: um estudo comparativo envolvendo gestores com diferentes perfis.

Uilcleides Braga da Silva, Adriano Leal Bruni, José Maria Dias Filho, Enoque Barbosa dos Santos

que não se enquadram nas características instrumentais do processo racional como, por exemplo, Excesso de Confiança, Efeito Framing.

A complexidade dos ambientes organizacionais (BUSHMAN; PIOTROSKI; SMITH, 2004, MILLER, 2004, BEZERRA; SILVA, 2011) tem exigido dos agentes (gestores) entendimento do processo decisório, ou seja, necessidade entender não só os aspectos objetivos, ligados a racionalidade, a lógica dos fatos, mas também os aspectos emocionais, comportamentais que influenciam no processo. Tais aspectos comportamentais agregam ao processo decisório a subjetividade no julgamento, ocasionando vieses cognitivos, levando a possíveis erros de julgamentos.

A mente humana ao lidar com situações que envolvam incertezas tende a simplificar ao máximo os problemas, criando alternativas que podem induzir a decisões errôneas. Estudos em finanças comportamentais (HOBSON; KACHELMEIER, 2005; SPRINGER; BORTHICK, 2007 e outros) apontam diversos tipos de heurísticas que conduzem a vieses que podem levar o tomador de decisão a erros graves: Representatividade; Disponibilidade; Ancoragem; Efeito Certeza; Aversão a Perda; Excesso de Confiança; entre outros.

As pesquisas voltadas para finanças comportamentais observam diversos tipos de vieses no processo decisório. Entre esses, em razão do problema da presente pesquisa, é válido destar o efeito Sunk cost.

Os custos perdidos são os custos que já ocorreram e não podem ser recuperados. Esses custos não são considerados relevantes, uma vez que já ocorreram e não devem influenciar na tomada de decisões futuras sob a abordagem das teorias econômicas.

Braverman e Barby (2012, p. 1) definem o efeito sunk cost como "tendência de seguir um curso de ação, mesmo depois que provou ser de qualidade inferior, porque os recursos foram investidos nesse curso de ação." Macaskill e Hackenberg (2012, p. 212) apontam que "o efeito sunk cost ocorre quando as pessoas persistem em um curso não-ideal de ação, porque eles já investiram tempo e recursos nele". São vieses que pessoas consideram ao continuar com um investimento mesmo sabendo que não terá os retornos esperados, somente pelo fato de já terem investido algum recurso e 
Decisões, complexidades, agentes e sunk costs: um estudo comparativo envolvendo gestores com diferentes perfis.

Uilcleides Braga da Silva, Adriano Leal Bruni, José Maria Dias Filho, Enoque Barbosa dos Santos

não observam qual seria a maneira mais viável e menos prejudicial para se tratar a situação.

Conforme Pavlic e Passino (2011, p. 58) a "observação do efeito sunk cost é uma intuição enigmatica, porque sugere que este comportamento é abaixo do ideal", pessoas continuam a aplicar recursos em um projeto mesmo sabendo que será em vão na tentativa de amenizar os possiveis resultados negativos. $O$ efeito do custo perdido foi observado nos estudos de Friedman et al. (2007) que em seus estudos encontraram evidências de sunk cost, constatando que erros teimosos são mais freqüentes quando os valores dos custos irrecuperáveis aumentam.

Wang e Yang (2010) em seus estudos examinaram que a manifestação do efeito sunk cost no comportamento de compra dos consumidores pode redefinir o preço entre as partes interessadas, evidenciando em seus resultados que o efeito sunk cost sobre uma taxa de adesão a uma empresa fornece incentivos para aumentar o preço unitário e /ou para aumentar a cobertura de mercado de taxas mais baixas de adesão. Esses autores acreditam que as preferencias de uma pessoa e as regras do jogo resultantes podem mudar quando a situação é percebida de forma diferente. Como exemplo eles citam que o sentimento dos consumidores pode ser diferente antes e depois da taxa de inscrição paga a uma empresa e, ainda, que a taxa de adesão pode não somente aumentar a fidelidade como também a demanda ex post, simplesmente pelo fato de já se ter efetuado o pagamento de uma taxa inicial de inscrição.

Buchheit e Feltovich (2011, p. 336) também discutem os paradoxos do efeito sunk cost e decisões racionais, concluindo que o sunk cost tem enfeito em forma de $\mathrm{U}$, ou seja, "de baixo para nível médio, diminuição de preços médios, mas a partir de níveis médios e altos, os preços aumentam a média" (tradução livre).

A pesquisa de Silva, Souza e Domingos (2008) teve como foco verificar a informação do valor investido num determinado projeto sobre o efeito sunk cost, encontrando como resultados que o tomador de decisão torna-se mais sensível ao se defrontar com um ambiente que envolve grandes volumes de recursos, mas em geral através do teste estatístico Qui-quadrado observaram que a evidenciação do valor do 
Decisões, complexidades, agentes e sunk costs: um estudo comparativo envolvendo gestores com diferentes perfis. Uilcleides Braga da Silva, Adriano Leal Bruni, José Maria Dias Filho, Enoque Barbosa dos Santos

custo perdido não influencia a decisão dos gestores de forma significativa. Outra pesquisa recente envolvendo o viés efeito sunk cost é o estudo de Segantini et al. (2011) que encontraram resultados parecidos, também não encontraram em suas investigações relação significativa entre a manifestação do viés e o valor do investimento.

\section{PROCEDIMENTOS METODOLÓGICOS}

A pesquisa assume como procedimento de coleta de dados a aplicação de questionário, contendo situações envolvendo efeito sunk cost com o objetivo de examinar como os gestores de entidades públicas e privadas tomam decisões em situações associadas ao mesmo, bem como questões que caracterizem o perfil dos respondentes.

É caracterizada como um quase-experimento, onde observou cuidadosamente o cenário apresentado na tentativa de aproximar ao máximo possível a situação ao ambiente real, para que os respondentes não fossem surpreendidos com situações muito aquém da realidade. Conforme Marcelino (2011, p. 53), o quase-experimento é "um estudo em que se manipula deliberadamente pelo menos uma variável independente para observar seu efeito e relação com uma ou mais variáveis dependentes, assim como nos experimentos puros". Nesse estudo não foi possível um tratamento experimental devido à necessidade de manipular valores para verificar a ocorrência do fenômeno sunk cost.

Os dados foram coletados em uma amostra composta por 128 alunos dos cursos de pós-graduação em Administração e Contabilidade da Universidade de Salvador (UNIFACS), Universidade do Estado da Bahia (UNEB) e Universidade Federal da Bahia (UFBA), e a aplicação dos questionários ocorreu em maio e junho de 2012. As perguntas que compunham os questionários visaram caracterizar a amostra, identificando variáveis como: idade, escolaridade, gênero, área de atuação, experiência na área profissional, para observar a influência dessas variáveis na percepção do efeito 
Decisões, complexidades, agentes e sunk costs: um estudo comparativo envolvendo gestores com diferentes perfis.

Uilcleides Braga da Silva, Adriano Leal Bruni, José Maria Dias Filho, Enoque Barbosa dos Santos

sunk cost nos julgamentos, bem como identificar quais respondentes tinham experiência em cargos de gestão e assim também verificar a relação entre essa variável e a ocorrência do viés.

O instrumento de pesquisa foi elaborado a partir de cenários adaptados dos estudos de Domingos (2007) e com base na pesquisa de Arkes e Blumer (1985) "Problema da companhia aérea". Foram utilizados dois tipos de questionários tipo A e tipo B. O questionário Tipo A apresentava uma situação envolvendo um custo irrecuperável no valor de 9 milhões de um investimento de 10 milhões em um projeto para desenvolvimento de uma vacina, onde o respondente teria que julgar se continuaria o investimento e correr o risco de perder mais 1 milhão, considerando que as chances de fracasso eram altas conforme cenário a seguir. Já o questionário tipo $B$ apresentava um custo irrecuperável de $50 \%, 5$ milhões, sendo uma decisão envolvendo mais 5 milhões.

Esses dois cenários diferentes permitiram verificar a sensibilidade dos gestores quanto aos valores investidos. Se estes julgam e tomam suas decisões de maneira mais lógica ou são afetados pelo viés do Efeito sunk cost e se acontece alguma variação entre os julgamentos de profissionais públicos e privados.

\section{ANÁLISE DOS DADOS}

No primeiro questionamento buscou-se obter dados relacionados com o perfil dos respondentes, mais especificamente sobre faixa etária, gênero e formação. Os dados sobre faixa etária e formação constam na Tabela 1. 
Decisões, complexidades, agentes e sunk costs: um estudo comparativo envolvendo gestores com diferentes perfis. Uilcleides Braga da Silva, Adriano Leal Bruni, José Maria Dias Filho, Enoque Barbosa dos Santos

Tabela1: Perfil dos respondentes

\begin{tabular}{ccc|ccc}
\hline \multicolumn{2}{c|}{ Idade } & & Formação & Fi & $\%$ \\
\hline Anos & $\mathbf{F i}$ & $\%$ & Qual sua formação? & 35 & 27,3 \\
$19-25$ & 18 & 14 & Ciências Contábeis & 33 & 25,8 \\
$26-30$ & 42 & 32,9 & Administração & 26 & 20,4 \\
$31-35$ & 34 & 26,5 & Marketing/ Relações Públicas/ Comunicação Social & 4 & 3,2 \\
$35-40$ & 14 & 10,9 & Tecnologia de Informação & 2 & 1,6 \\
41 ou mais & 15 & 11,9 & Economia/ Estatística & 22 & 17,5 \\
Não responderam & 5 & 3,8 & Outros & 6 & 4,2 \\
\hline Total & $\mathbf{1 2 8}$ & $\mathbf{1 0 0}$ & Não responderam & $\mathbf{1 2 8}$ & $\mathbf{1 0 0}$ \\
\hline
\end{tabular}

Fonte: Dados da pesquisa (2012).

É possível verificar na Tabela 1 que mais de $70 \%$ dos respondentes tem entre 26 e 40 anos de idade. Quanto ao gênero a amostra é composta por 66 mulheres e 62 homens, e destes respondentes $53,1 \%$ tem formação em áreas gerenciais como contabilidade ou administração, ou seja, podem ter algum conhecimento técnico quanto ao processo de decisão, enquanto 20,4\% apresentam formação em Marketing, Relações Públicas ou Comunicação Social. Na categoria outros, existem respondentes formados em diversas áreas como Engenharia Elétrica, Serviço Social, Odontologia, Enfermagem, Turismo, Jornalismo e outros, não voltados para funções gerenciais.

No que se refere ao tempo de experiência e área de atuação, os dados obtidos são apresentados na Tabela 2. 
Decisões, complexidades, agentes e sunk costs: um estudo comparativo envolvendo gestores com diferentes perfis. Uilcleides Braga da Silva, Adriano Leal Bruni, José Maria Dias Filho, Enoque Barbosa dos Santos

Tabela 2: Experiência Profissional

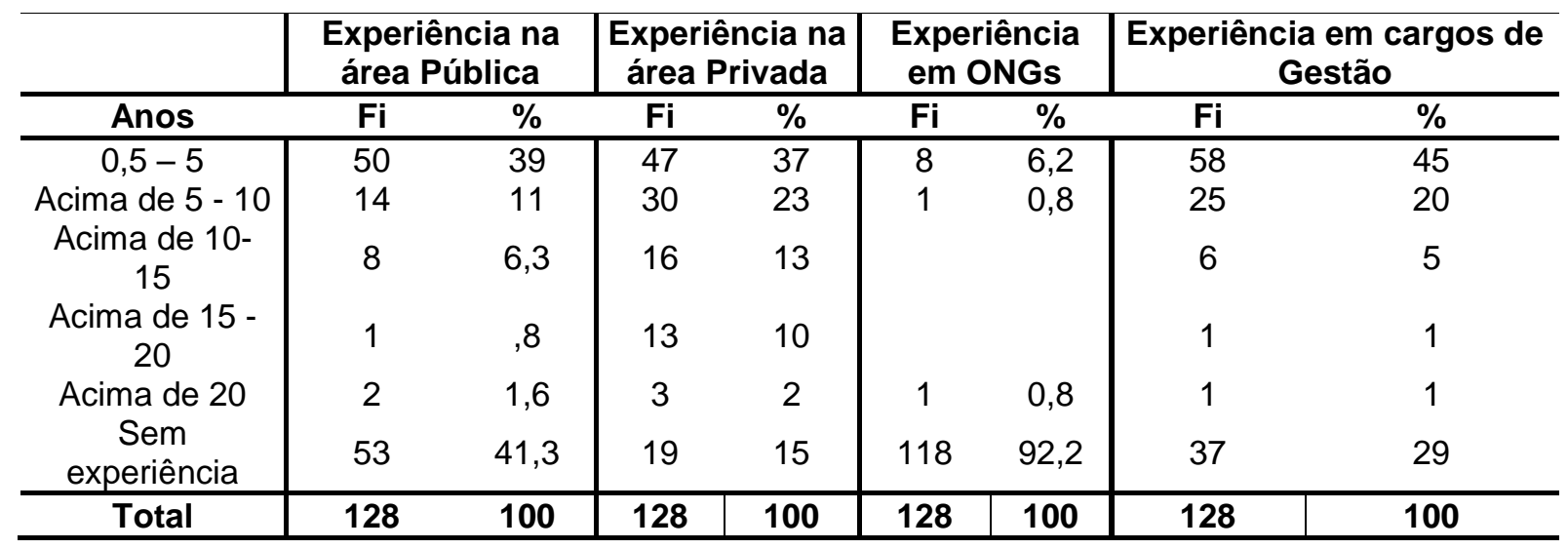

Fonte: Dados da pesquisa (2012).

Os dados expostos na Tabela 2 permitem observar que 39\% dos 128 participantes tem até 5 anos de experiência de atividades na área pública, $11 \%$ tem de 5 a 10 anos de experiência, 8,7 \% mais de 10 anos e 41,3\% não tem experiência de atuação na área pública. No setor privado, $37 \%$ dos 128 participantes tem até 5 anos de experiência, 23\% tem de 5 a 10 anos de experiência, $25 \%$ mais de 10 anos e somente 15\% não tem experiência de atuação na área privada. Com experiência de atuação em Organizações do Terceiro Setor - ONGs são encontrados somente 7,8\% dos respondentes.

A maioria dos respondentes tem experiência em cargos de gestão, sendo que $71 \%$ apresentam experiência em cargos que envolvem processos decisórios, o que pode influenciar na percepção do efeito sunk cost investigado mais a frente.

$\mathrm{Na}$ Tabela 3 constam os dados relacionados com a manifestação do efeito sunk cost. 
Decisões, complexidades, agentes e sunk costs: um estudo comparativo envolvendo gestores com diferentes perfis. Uilcleides Braga da Silva, Adriano Leal Bruni, José Maria Dias Filho, Enoque Barbosa dos Santos

Tabela 3: Manifestação do Efeito sunk cost.

\begin{tabular}{|c|c|c|c|c|c|c|}
\hline \multicolumn{7}{|c|}{ Investiria? } \\
\hline & \multicolumn{2}{|c|}{$\begin{array}{l}\text { Cenário I- Sunk cost- } \$ 5 \\
\text { milhões }\end{array}$} & \multicolumn{2}{|c|}{ Cenário II- Sunk cost- \$ 9 milhões } & \multicolumn{2}{|c|}{ Total } \\
\hline & $\mathbf{F i}$ & $\%$ & $\mathrm{Fi}$ & $\%$ & Fi Total & $\%$ \\
\hline Não & 20 & 31 & 22 & 34,4 & 42 & 33 \\
\hline Sim & 44 & 69 & 42 & 65,6 & 86 & 67 \\
\hline $\begin{array}{c}\text { Tota } \\
\text { I }\end{array}$ & 64 & 100 & 64 & 100 & 128 & 100 \\
\hline
\end{tabular}

Fonte: Dados da pesquisa (2012).

Os dados expostos na Tabela 3 indicam a ocorrência do efeito sunk cost, uma vez que $67 \%$ dos respondentes assinalaram que continuariam o investimento, enquanto que $33 \%$ foram mais conservadores e abortariam o projeto.

Considerando apenas a análise estatística descritiva, constata-se que os dados se mostram contrários ao esperado. Em se tratando da sensibilidade dos respondentes em relação ao aumento no valor de custos irrecuperáveis, observa-se que ocorre uma pequena redução das respostas afirmativas $(69 \%)$ no cenário I (com sunk cost no valor de 5 milhões) para 65,6\% no cenário II (com sunk cost no valor de 5 milhões). Assim, percebe-se que o valor do sunk cost não influencia de forma significativa a tomada de decisão.

A pesquisa corrobora resultados do estudo de Silva, Souza e Domingos (2008). Segantini, Vieira e Silva (2011) observaram que o valor influencia na persistência em investimento, aumentando a sensibilidade.

A fim de verificar a dificuldade encontrada pelos respondentes para decidir sobre continuar ou interromper o investimento, foi apresentada uma escala com variação de 1 a 7, onde 1 representa maior grau de facilidade enquanto 7 maior grau de dificuldade conforme Tabela 4. A Tabela 4 mostra também o escore para a questão que verifica a fidedignidade utilizando intervalos entre 1 e 7 referente a compreensão do cenário, facilidade de ocorrência da situação e realismo apresentado no instrumento de coleta, onde 1 significa "discordar totalmente" e 7 "concordar totalmente". 
Decisões, complexidades, agentes e sunk costs: um estudo comparativo envolvendo gestores com diferentes perfis.

Uilcleides Braga da Silva, Adriano Leal Bruni, José Maria Dias Filho, Enoque Barbosa dos Santos

Tabela 4: Grau percebido de dificuldade e fidedignidade do cenário

\begin{tabular}{|c|c|c|c|c|c|c|c|c|c|c|}
\hline & \multicolumn{5}{|c|}{$\$ 5$ milhões } & \multicolumn{3}{|c|}{ \$9 milhões } & \multicolumn{2}{|c|}{ Total } \\
\hline & \multicolumn{2}{|r|}{$\mathbf{F i}$} & \multicolumn{3}{|c|}{$\%$} & $\mathbf{F i}$ & \multicolumn{2}{|c|}{$\%$} & Fi Total & $\%$ \\
\hline Muito fácil (1) & \multicolumn{2}{|r|}{4} & \multicolumn{3}{|c|}{6} & 3 & \multicolumn{2}{|l|}{5} & 7 & 5 \\
\hline Fácil (2-3) & \multicolumn{2}{|r|}{15} & \multicolumn{3}{|c|}{23} & 14 & \multicolumn{2}{|c|}{22} & 29 & 23 \\
\hline Normal (4) & \multicolumn{2}{|r|}{10} & \multicolumn{3}{|c|}{16} & 18 & \multicolumn{2}{|c|}{28} & 28 & 22 \\
\hline Difícil (5-6) & \multicolumn{2}{|r|}{32} & \multicolumn{3}{|c|}{50} & 25 & \multicolumn{2}{|c|}{39} & 57 & 45 \\
\hline Muito difícil (7) & \multicolumn{2}{|r|}{3} & \multicolumn{3}{|c|}{5} & 4 & \multicolumn{2}{|l|}{6} & 7 & 5 \\
\hline Total & \multicolumn{2}{|r|}{64} & \multicolumn{3}{|c|}{100} & 64 & \multicolumn{2}{|c|}{100} & 128 & 100 \\
\hline Fidedignidade & $\begin{array}{l}N \\
64\end{array}$ & $\begin{array}{l}\text { Mín. } \\
2,33\end{array}$ & $\begin{array}{c}\text { Máx } \\
7\end{array}$ & $\begin{array}{c}\text { Média } \\
4,661 \\
5 \\
\end{array}$ & $\begin{array}{c}\text { Desvio } \\
\text { Padrão } \\
1,1585\end{array}$ & $\begin{array}{l}\mathbf{N} \\
64\end{array}$ & $\begin{array}{c}\text { Mín. } \\
2\end{array}$ & $\begin{array}{c}\text { Máx } \\
7\end{array}$ & \begin{tabular}{|c|} 
Média \\
4,757 \\
8 \\
\end{tabular} & $\begin{array}{l}\text { Desvio } \\
\text { Padrão } \\
1,20146\end{array}$ \\
\hline $\begin{array}{c}\text { Total } \\
\text { Respondentes }\end{array}$ & \multicolumn{5}{|c|}{64} & \multicolumn{4}{|c|}{64} & 128 \\
\hline
\end{tabular}

Fonte: Dados da pesquisa (2012).

Encontrou - se uma média de 4.6615, observando que em média os respondentes concordam razoavelmente com o cenário, sendo esse instrumento fidedigno e entendível pelos respondentes como uma situação razoavelmente comum.

Para confirmar a ocorrência do efeito, analisaram-se as respostas dos dois cenários através do teste estatístico do Qui - quadrado $\left(X^{2}\right)$, Tabela 5. 
Decisões, complexidades, agentes e sunk costs: um estudo comparativo envolvendo gestores com diferentes perfis. Uilcleides Braga da Silva, Adriano Leal Bruni, José Maria Dias Filho, Enoque Barbosa dos Santos

Tabela 5: Testes de associação em relação à manifestação do fenômeno

\begin{tabular}{c|cccccc|ccc}
\hline \multicolumn{1}{c}{ Valor do sunk cost } & \multicolumn{6}{c}{ Estatísticas descritivas } & \multicolumn{2}{c}{ Teste t para Igualdade de } \\
Médias
\end{tabular}

Fonte: Dados da pesquisa (2012).

Observa-se uma concentração significativa de freqüências na resposta "Sim", o que caracteriza a manifestação do viés nos dois cenários (sunk costs iguais a $\$ 5$ milhões e $\$ 9$ milhões). No cenário I, mais de $60 \%$ dos respondentes escolheram a opção "sim", afirmando continuar o investimento, observando $X^{2}=9.921$ e 0.002 de significância. Já no cenário II ocorreu uma pequena variação tendo um $X^{2}=6.250 \mathrm{e}$ 0.012 de significância, não significativa da ocorrência o viés, verificada na variação do $X^{2}$. Logo, o seu quase-experimento consegue identificar o fenômeno.

Também se verificou a associação entre a manifestação do viés e características dos respondentes como: tempo de experiência profissional; experiência profissional em 
Decisões, complexidades, agentes e sunk costs: um estudo comparativo envolvendo gestores com diferentes perfis.

Uilcleides Braga da Silva, Adriano Leal Bruni, José Maria Dias Filho, Enoque Barbosa dos Santos

cargos de gestão; tempo de vinculação profissional a entidades públicas ou privadas, idade e gênero. Foi realizado o teste $t$ para verificar as diferenças das respostas quanto a manifestação do viés, bem como associação do viés e outras variáveis (Tabela 5).

Não foram encontradas diferenças estatisticamente significativas entre os julgamentos de continuar o investimento ou abortá-lo e o variável tempo de experiência no setor privado (significância de 0, 4193 para o cenário I e 0, 2006 para o cenário II).

Os resultados encontrados não confirmam o esperado a partir de situações hipotéticas, de que o simples fato de uma pessoa apresentar vivencia em empresas do setor privado influencia na ocorrência do viés. Sendo assim, esta variável não explica a ocorrência do viés.

Quanto à variável gênero, foi possível verificar que no geral esta não é significativa, embora tenha sido significativa quando relacionada ao cenário I com significância de 0,2341 , o que não acontece quando aumenta o valor do sunk cost, quando a significância altera para 0,7221 , o que significa que não existe de modo geral uma associação significativa entre a ocorrência do viés e o gênero. A variável idade também não aparenta ser significante.

Esses resultados encontrados em relação às variáveis idade e gênero corroboram com os resultados encontrados por Rover et al. (2009). Contudo, não acontece o mesmo com os resultados da pesquisa de Domingos (2007), que encontrou que tanto a idade quanto a formação apresentaram variações significativas em relação ao viés.

Observa-se ainda na Tabela 5, que o fato de se ter experiência em cargos de gestão não elimina a persistência e manifestação do viés, conforme poderia se imaginar inicialmente. Logo, a hipótese de que os profissionais com experiência em cargos de gestão estão menos propensos ao efeito sunk cost não foi confirmada pelos testes estatísticos. A experiência em cargos de gestão não está associada ao julgamento de investir ou não o saldo remanescente de um orçamento de um projeto perdido. A hipótese da experiência em setor privado ser observada na manifestação do efeito sunk cost não foi confirmada, e os respondentes aparentam ter julgamentos uniformes em relação ao sunk cost. 
Decisões, complexidades, agentes e sunk costs: um estudo comparativo envolvendo gestores com diferentes perfis.

Uilcleides Braga da Silva, Adriano Leal Bruni, José Maria Dias Filho, Enoque Barbosa dos Santos

Domingos (2007) acredita que variáveis como: a responsabilidade pessoal, probabilidade de sucesso, experiência com conceitos econômicos, tipo de feedback negativo, variáveis culturais, entre outras, podem influenciar na ocorrência do efeito sunk cost.

\section{CONSIDERAÇÕES FINAIS}

Sob o prisma do efeito sunk cost, os resultados da pesquisa evidenciam a ocorrência do mesmo, indicando que na pratica os custos perdidos não são irrelevantes no processo decisório, tendo se verificado a ocorrência do efeito sunk cost em quase $70 \%$ das respostas. Não foram encontradas evidências significativas de que o valor do custo perdido influencia a tomada de decisão.

Também não foram encontradas diferenças estatisticamente significativas entre a ocorrência do viés e a variável tempo de experiência no setor privado. Sendo assim, a hipótese de que a experiência em setor privado torna os profissionais menos propensos à manifestação do efeito sunk cost não foi confirmada, não sendo rejeitada a hipótese nula de que a experiência em setor privado não influencia a manifestação do efeito sunk cost. Não foram encontradas associações significativas entre as variáveis: gênero e idade e a ocorrência do viés. A variável gênero igualmente não foi significativa.

Considerando a hipótese de que "os profissionais com experiência em cargos de gestão são menos sensíveis, propensos ao efeito sunk cost" por lidar com situações que envolvam decisões, os resultados encontrados mostraram que não existem evidencias relevantes entre o fato de uma pessoa ser experiente em cargos que envolvam decisões e a manifestação do recurso. $O$ fato de se ter experiência em cargos de gestão não elimina a persistência e manifestação do viés, conforme seria imaginado inicialmente, sendo a hipótese refutada.

Sugere-se que esta pesquisa seja replicada considerando uma amostra maior. $\mathrm{O}$ questionário poderia conter mais cenários para serem comparados e, ainda, poderia ter questões discursivas onde os respondentes poderiam justificar o motivo de continuar ou 
Decisões, complexidades, agentes e sunk costs: um estudo comparativo envolvendo gestores com diferentes perfis.

Uilcleides Braga da Silva, Adriano Leal Bruni, José Maria Dias Filho, Enoque Barbosa dos Santos

não um investimento em situações de ocorrência de sunk cost. Isso permitiria que através de uma análise de discurso se verificasse as motivações nos dois setores, tanto público quanto privado, e compará-las entre esses setores a fim de verificar se existe diferença entre eles.

\section{REFERÊNCIAS}

ALRABADI, Dima W. GHARAIBEH, Mohammad A. ZURIGAT, Ziad, M. (2010) WhatMakes Investors Overconfident? Evidence from Amman Stock Exchange. European Journal of Economics, Finance and Administrative Sciences. ISSN 1450-2275 Issue 43.

BEZERRA, Douglas Moraes, SILVA, F. A. G. (2011) Abordagem multiparadigmática em Administração: a busca de uma melhor compreensão da complexidade organizacional. Revista Espaço Acadêmico. № 124, set.

BRAVERMAN, Jennifer A; BARBY, J.S. Blumenthal. (2012). Assessment of the sunkcost effect in clinical decision-making. Social Science \& Medicine. P.1-7.

BRUNI, A. L. et al. (2010). A presença do excesso de confiança em práticas orçamentárias: Um estudo experimental. In: IV Congresso ANPCONT.

BUCHHEIT, S. e FELTOVICH, N. (2011). Experimental evidence of a sunk-cost paradox: a study of pricing behavior in Bertrand-edgeworth duopoly. International Economic Review. Vol. 52, No. 2, May.

BUSHMAN, R.; PIOTROKI, J. SMITH, A. (2004). What Determines Corporate Transparency? Journal of Accounting Research. Vol. 42 No. 2 May.

CAVALHEIRO, Everton Anger. (2010). Tolerância ao Risco: Uma Análise sob a Ótica Comportamental. (Dissertação de Mestrado). Universidade Federal de Santa Maria (RS),116 p.

DOMINGOS, Naiára. (2007). Custos perdidos e insistência irracional. Brasília. Dissertação de Mestrado. Universidade Federal de Pernambuco e Universidade Federal do Rio Grande do Norte. Brasília. 126 f. 
Decisões, complexidades, agentes e sunk costs: um estudo comparativo envolvendo gestores com diferentes perfis. Uilcleides Braga da Silva, Adriano Leal Bruni, José Maria Dias Filho, Enoque Barbosa dos Santos

FRIEDMAN et al. (2007). Searching for the sunk cost fallacy. Published online: Economic Science Association.

HOBSON, Jessen L, KACHELMEIER, Steven J. Strategic. (2005). Disclosure of Risky Prospects: A Laboratory Experiment. The Accounting Review. Vol. 80, No. 3. pp. 825846.

MACASKILL, Anne C.; HACKENBERG, Timothy D. (2012). Providing a reinforcement history that reduces the sunk cost effect. Behavioural Processes. 89. 212-218.

MARCELINO, Carolina Venturini. (2011) Viés da cognição numérica e práticas orçamentárias: um estudo quase-experimental. (Dissertação de Mestrado). Programa de Pós-Graduação em Ciências Contábeis, p. 135. Salvador- Ba.

MILLER, G. S. (2004). Discussion of What Determines Corporate Transparency? Journal of Accounting Research. Vol. 42 No. 2, may, 2004.

PAVLIC, Theodore P., PASSINO, Kevin M. (2010). The Sunk-Cost Effect as an Optimal Rate-Maximizing Behavior. Published online: Springer Science Business Media B.V.: 24 July.

ROVER, Suliani et al. (2009). Efeito Sunk costs: O Conhecimento Teórico Influencia no Processo Decisório de Discentes? Brazilian Business Review. Vol. 6, No.3, p. 247-263 Set -Dez.

SEGANTINI, G. T. et al. (2011). Efeito sunk costs: avaliação da influência do custo perdido no processo de tomada de decisão dos gestores das empresas de construção civil. $V$ congresso Anpcont, Vitoria-ES.

SILVA, C. A. T.; LIMA, D. H. S. (2007). Formulation Effect: Influencia da Forma de Apresentação sobre o Processo Decisório de usuários de informações financeiras. In: Encontro da Associação Nacional de Programas de Pós-Graduação em Administração EnAnpad, XXXI, Rio de Janeiro. Disponível: < http://pt.scribd.com/doc/54510977/CONA2290TC>. Acesso em 15/maio/2012.

SILVA, C. A. T; SOUZA, F. A.; DOMINGOS, N. T. (2008). Efeito Do Custo Perdido: A Influência do Custo Perdido na Decisão de Investimento. RCO - Revista de Contabilidade e Organizações. FEARP/USP, v. 2, n. 2, p. 87 - 99 jan./abr. 
Decisões, complexidades, agentes e sunk costs: um estudo comparativo envolvendo gestores com diferentes perfis. Uilcleides Braga da Silva, Adriano Leal Bruni, José Maria Dias Filho, Enoque Barbosa dos Santos

SPRINGER, Carol W., BORTHICK, A. Faye. (2007). Improving Performance in Accounting: Evidence for Insisting on Cognitive Conflict Tasks. Issues In Accounting Education, Vol. 22, No. 1, pp. 1-19, February.

VA, José Carlos. (2006). A delicada disputa pelas cabeças na evolução do serviço público no Brasil pós-redemocratização. Cadernos ENAP. Edição especial.

VAZ, J. Carlos, LOTTA, G. S. (2011). A contribuição da logística integrada às decisões de gestão das políticas públicas no Brasil. Revista de Administração Pública. Rio de Janeiro 45(1):107-39, jan./fev.

WANG, X. Henry e YANG, Bill Z. (2010). The sunk-cost effect and optimal two-part pricing. The journal of Economics. Volume 101, Number 2, p.133-148.

WATT, Richard, VÁZQUEZ, Francisco J. (2012). The Effect of Prices on Risk Aversion. Theoretical Economics Letters, 2, 40-44 doi:10.4236/tel.2012.21007 Published Online February 2012 (http://www.SciRP.org/journal/tel).

Data de Submissão: 24/05/2013

Data de Aceite: 20/05/2014 